\title{
Calcified decellularized arterial scaffolds impact vascular smooth muscle cell transformation via downregulating $\alpha$-SMA expression and upregulating OPN expression
}

\author{
HUAPING XIN $^{1 *}$, ZHIMIN WANG ${ }^{2 *}$, SHUWU WU $^{1}$, PENG WANG $^{2}$, \\ XIAOXIAO TAO ${ }^{2},{\text { CHENHUA } \mathrm{XU}^{2} \text { and LILING YOU }}^{2}$ \\ ${ }^{1}$ Department of Geriatrics, The People's Hospital of Yichun City, Yichun, Jiangxi 336000; \\ ${ }^{2}$ Department of Neurology, Taizhou First People's Hospital, Taizhou, Zhejiang 318000, P.R. China
}

Received March 14, 2018; Accepted March 26, 2019

DOI: $10.3892 /$ etm.2019.7626

\begin{abstract}
The underlying mechanisms of arterial remodeling (AR) remain unclear. Studies have indicated that decellularized scaffolds stimulate the differentiation of fibroblasts into myofibroblasts and promote the accumulation of the extracellular matrix (ECM). In the present study, the impact of ECM changes following AR on vascularsmooth musclecell(VSMC) phenotypes was investigated. VSMCs were co-cultured with normal or calcified decellularized arterial scaffolds. The expression levels of $\alpha$-smooth muscle actin ( $\alpha$-SMA) and osteopontin (OPN) were measured at 2,5, 10,15 and 21 days following the establishment of the co-culture systems. The expression of $\alpha$-SMA in the normal co-culture group was significantly increased compared with that in the calcified arterial decellularized scaffold co-culture group $(\mathrm{P}<0.05$ and $\mathrm{P}<0.001)$. In addition, the expression of OPN in the AR co-culture group was significantly increased compared with the normal co-culture group $(\mathrm{P}<0.05$ and $\mathrm{P}<0.001)$. To conclude, the calcified decellularized arterial scaffolds impact VSMC transformation by downregulating $\alpha$-SMA expression and upregulating OPN expression $(\mathrm{P}<0.001)$. To the best of our knowledge, the present study is the first study that co-cultured VSMCs with normal or calcified decellularized arterial scaffolds.
\end{abstract}

\section{Introduction}

Vascular calcification increasingly affects the aging and dysmetabolic population worldwide $(1,2)$. Vascular

Correspondence to: Dr Liling You, Department of Neurology, Taizhou First People's Hospital, 218 Hengjie Road, Huangyan, Taizhou, Zhejiang 318000, P.R. China

E-mail: yl11025@163.com

*Contributed equally

Key words: arterial remodeling, extracellular matrix, decellularized arterial scaffolds, vascular smooth muscle cell, vascular calcification, osteopontin calcification, which occurs in the intima and media of the artery, is considered an independent risk factor of cardiovascular and cerebrovascular diseases (1). Although angiotensin-converting enzymes and statins can stabilize atherosclerosis, they cannot reverse vascular calcification $(3,4)$. The molecular complexity involved in the regulation of vascular calcification is still unclear $(4,5)$. Notably, the arterial remodeling (AR) process serves an important role in the pathogenesis of vascular calcification (6).

The AR process includes smooth muscle cell (SMC) migration and proliferation, and extracellular matrix (ECM) production (7). During the AR process, the phenotypic transformation of vascular smooth muscle cells (VSMCs) causes abnormal development of the ECM in blood vessels (7). Studies have indicated that elevated extracellular calcium and phosphate levels impact the survival and phenotype of VSMCs, which results in cellular adaptations and damage that ultimately promotes calcification (8). As a result, the normal contractile SMCs are converted into synthetic SMCs (9), with significantly decreased $\alpha$-SMA expression and significantly increased OPN expression (10).

Little is known regarding how the alterations in ECM composition and VSMCs impact vascular calcification, even though $>50 \%$ of the neointimal hyperplasia consists of ECM proteins (7). Furthermore, the mechanisms of VSMC activation in the AR process remain unclear. Studies have indicated that VSMCs can be activated by persistent injury or other factors, including the renin-angiotensin aldosterone system (11), transforming growth factor- $\beta$ (12), connective tissue growth factor (13) and matrix metalloproteinases (14). In addition, the change in the proportion of ECM components of the vascular wall is also vital for the transformation of the VSMC phenotype (9).

ECM is a complex mixture of structural and functional proteins, glycoproteins, and proteoglycans arranged in a unique, tissue specific three-dimensional (3D) ultrastructure (15). The natural 3D spatial structure provides a convenient platform for cells to regenerate (16). Similar to the native ECM (17-19), decellularized scaffolds are completely depleted of cells and their components by chemical or physical methods, but preserve the majority of the 3D spatial structures 
of tissues and organs (20). The decellularized scaffolds have a good biological capacitance, low immunogenicity, and are widely used in the field of tissue engineering (21). Due to the complexity of the ECM components and the spatial structure of the vascular wall, it is difficult to elucidate the impact of the ECM on VSMC phenotypic transformation following artery reconstruction in vitro $(22,23)$. Therefore, in the current study, decellularized vascular scaffolds were used to determine whether ECM could impact the phenotypic transformation of VSMCs following AR in vascular calcification.

\section{Materials and methods}

Rat model of vascular calcification. The rat model of arterial calcification was constructed as described previously (17). A total of 22 male 6-week-old Sprague Dawley rats (Animal Experimental Center of Wenzhou Medical University, Wenzhou, China) with mean body weight of $\sim 180 \mathrm{~g}$ were used to evaluate the effects of arterial calcification. Animals were maintained at a controlled temperature $\left(22 \pm 2^{\circ} \mathrm{C}\right)$ and humidity $(55 \pm 5 \%)$ with a 12 -h light/dark cycle under specific-pathogen-free conditions with free access to food and water. Following a week acclimation period, 22 rats were divided into the arterial calcification group (experimental group; $\mathrm{n}=16$ ) and normal arterial group (control group; $n=6$ ). The animals in the arterial calcification group were injected with vitamin K (Chengdu Bite Pharmaceutical Group Co., Ltd.) $11.5 \mathrm{mg} /(100 \mathrm{~g}$ body weight/day) subcutaneously from the first day to the sixth, on the third day, subcutaneous injection of vitamin D3 (Beijing Solarbio Science \& Technology Co., Ltd.; 30,000 U/(100 g body weight/day) was continued once per day until the fifth day, and warfarin (Sangon Biotech Co., Ltd.) $150 \mathrm{mg} / \mathrm{kg}$ was administered twice per day orally until the sixth day; and normal arterial group were injected with $0.9 \%$ saline of the same volume with the same method. All rats were anesthetized with $300 \mathrm{mg} / \mathrm{kg} \mathrm{10 \%} \mathrm{chloral} \mathrm{hydrate} \mathrm{(cat.} \mathrm{no.}$ A600288; Sangon Biotech Co., Ltd., Shanghai, China) and euthanized by cervical dislocation on the 7th day following the model establishment. Following sacrifice, the rats were immersed in $75 \%$ alcohol for $5 \mathrm{~min}$ at room temperature. The thoracic cavity of the rats was opened on a sterile clean bench. The thoracic aorta and abdominal aorta were removed as soon as possible, and the residual blood clots were washed with PBS at $4^{\circ} \mathrm{C}$ three times, and PBS was exchanged every $5 \mathrm{~min}$. A small section of each segment of the vessels (the normal and the calcifiated vessels) was fixed with $4 \%$ paraformaldehyde for $\sim 30 \mathrm{~min}$ at room temperature using for Von Kossa staining. The remaining samples were dried using a Drying Oven (model DHG-9023A) for $15 \mathrm{~min}$ and stored in at $-20^{\circ} \mathrm{C}$ until use.

Von Kossa staining. Aortic vessels were examined by Von Kossa staining as follows: The sections (8- $\mu \mathrm{m}$-thick) were attached to a glass slide in a $37^{\circ} \mathrm{C}$ water bath, and dried at $37^{\circ} \mathrm{C}$ for $30 \mathrm{~min}$. The sections were incubated with $1 \%$ silver nitrate for $1 \mathrm{~h}$ at room temperature under a UV lamp, then rinsed with distilled water $>3$ times, washed off the black material on the surface with distilled water, then incubated with $5 \%$ sodium thiosulfate at room temperature for $2 \mathrm{~min}$. Samples were subsequently counterstained with $1 \%$ neutral red at room temperature for $10 \mathrm{~min}$ and images were captured under a fluorescent microscope (Olympus Corporation).

Preparation of aortic decellularized biological scaffold. Half of the normal and calcified blood vessels were continuously perfused (perfusion pressure, $3.6 \mathrm{mmHg}$ ) with $0.1 \%$ trypsin solution and $0.5-1 \%$ SDS solution at $37^{\circ} \mathrm{C}$ for $12 \mathrm{~h}$. The decellularization solution (trysin and SDS) was refreshed every $6 \mathrm{~h}$. The color and shape of the blood vessels at various time points $(0,30,60,120,180$ and $240 \mathrm{~min})$ were observed during perfusion in vitro. When the vessels became clean and transparent, the decellularization solution was replaced with $0.9 \%$ saline at room temperature for $1 \mathrm{~h}$ before use.

To confirm that decellularized scaffolds were successfully obtained, the DNA band in the normal arterial and calcified arterial decellularized scaffolds was detected by agarose gel electrophoresis. Total DNA was purified from normal arterial decellularized or non-decellularized scaffolds and calcified arterial decellularized or non-decellularized scaffolds, using a Mammalian genomic DNA extraction kit (Beyotime Institute of Biotechnology). Extracted DNA was applied to $1 \%$ agarose gel $100 \mathrm{~V}$ for $1 \mathrm{~h}$ at room temperature. The gel with DNA fragments was visualized and analyzed on the ChemiDoxTM XRS+ system (Bio-Rad Laboratories, Inc.).

Co-culture conditions. Direct co-cultures were established by adding VSMCs to normal arterial decellularized scaffolds or calcified arterial decellularized scaffolds in 24-well plates. Briefly, normal and calcified arterial decellularized scaffolds were perfused with $2 \%$ agarose solution at $37^{\circ} \mathrm{C}$, chilled at $4^{\circ} \mathrm{C}$ and sectioned into $5 \times 5 \mathrm{~mm}$ squared sections. Following removal of agarose, the arterial decellularized scaffolds were stored in PBS at $4^{\circ} \mathrm{C}$. VSMCs (A-10; American Type Culture Collection, Manassas, VA, USA) were suspended at a concentration of $1 \times 10^{7} / \mathrm{ml}$. A total of $10 \mu \mathrm{l}$ of cell suspension was added onto the arterial decellularized scaffold slices and incubated for $30 \mathrm{~min}$ at room temperature. The slices were transferred to 24-well plates. Cells were cultured in high-glucose Dulbecco's modified Eagle medium (Sigma-Aldrich; Merck KGaA) supplemented with $10 \%$ fetal bovine serum (Royacel) at $37^{\circ} \mathrm{C}$ in an incubator containing $5 \% \mathrm{CO}_{2}$ for 9 days. The media were changed every 3 days. Each co-culture experiment was performed three times. The mixed culture system of VSMC-arterial decellularized scaffolds was constructed. Specimens were obtained at 2, 5, 10, 15 and 21 days following co-culture. Subsequently, the expression of $\alpha$-SMA and OPN were detected.

Western blot analysis. The assay was performed using the following primary antibodies: Rabbit polyclonal anti- $\alpha$-SMA (cat. no. ab72583; Abcam), anti-OPN (cat. no. ab8448; Abcam), and anti-GAPDH (cat. no. AG019; Beyotime Institute of Biotechnology). Glass plates $(1.5 \mathrm{~mm})$ were washed and dried for use. Total protein was extracted with RIPA and was quantified using a BCA Protein Assay kit (Beyotime Institute of Biotechnology) and $30 \mu \mathrm{g}$ protein/lane was separated via SDS-PAGE on a $10 \%$ gel. After electrophoresis, proteins on the gel were transferred to a PVDF membrane, which was 
A

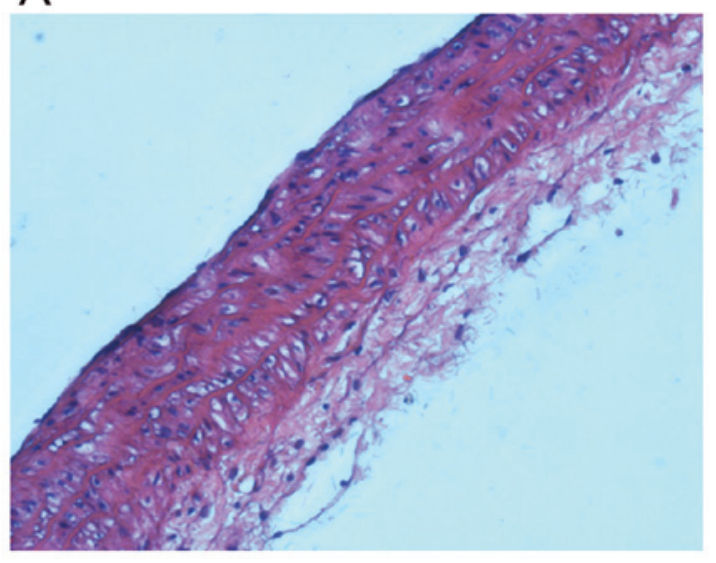

B

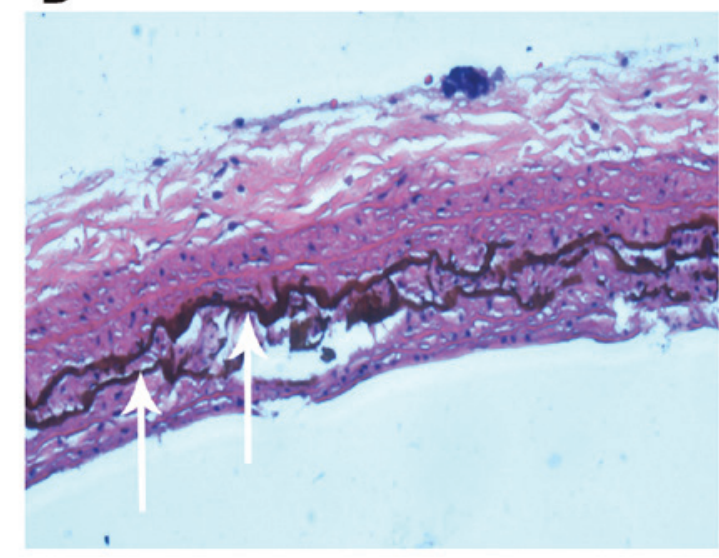

Figure 1. Von Kossa staining of aortas. (A) Von Kossa staining revealed no calcification in the thoracic aorta of normal rats (magnification, x40). (B) There were strongly positively stained black calcified nodules (white arrows) present in the thoracic aorta of rats with vascular calcification (magnification, $\mathrm{x} 40$ ).

incubated with $5 \%$ skim milk powder for $1 \mathrm{~h}$ at room temperature. All primary antibodies were incubated overnight at $4^{\circ} \mathrm{C}$, with a dilution ratio of 1:1,000, and then washed with TBST 3 times (5 min each time). Secondary antibody (HRP-labeled Goat Anti-Rabbit IgG; 1:2,000; cat. no. A0208; Beyotime Institute of Biotechnology) was added for $1 \mathrm{~h}$ at room temperature, then samples were washed 3 times with TBST. Visualization was performed with ECL (BeyoECL Star kit, Beyotime Institute of Biotechnology) for 2-5 min followed by exposure to a chemiluminescence imager (ChemiDoc ${ }^{\mathrm{TM}}$ $\mathrm{XRS}^{+}$; Bio-Rad Laboratories, Inc.).

Reverse transcription-quantitative polymerase chain reaction (RT-qPCR) detection of $\alpha-S M A$ and OPN. Total cellular RNA from VSMC co-cultured with scaffolds was purified using TRIzol reagent (Thermo Fisher Scientific, Inc.), and complementary DNA (cDNA) was synthesized using a ReverTra Ace ${ }^{\circledR}$ qPCR RT kit (Toyobo Life Science) at $37^{\circ} \mathrm{C}$ for $15 \mathrm{~min}$ and at $95^{\circ} \mathrm{C}$ for $5 \mathrm{~min}$. cDNA samples were placed immediately on ice and transferred to $-20^{\circ} \mathrm{C}$ prior to subsequent qPCR detection. The following $10-\mu 1$ reaction system was used for qPCR: $0.1 \mu \mathrm{l}$ cDNA template, $0.2 \mu \mathrm{l}$ of each primer, $4.5 \mu \mathrm{l}$ of $0.1 \%$ DEPC water and $5 \mu \mathrm{l}$ of SYBR Green qPCR premix (Takara Biotechnology Co., Ltd.). The following thermocycling conditions were used: Initial denaturation at $95^{\circ} \mathrm{C}$ for $30 \mathrm{sec}$; 40 cycles of $95^{\circ} \mathrm{C}$ for $5 \mathrm{sec}$ and $60^{\circ} \mathrm{C}$ for $30 \mathrm{sec}$. Using GAPDH as an internal reference gene, $2^{-\Delta \Delta \mathrm{Cq}}$ was used to calculate the expression level of each gene of interest (24). The following primer sequences were used: $\alpha$-SMA forward, 5'-AGGGAC TAATGGTTGGAATGG-3' and reverse, 5'-CAATCTCAC GCTCGGCAGTAG-3'; OPN, forward, 5'-CTGATGCTACAG ACGAGGAC-3' and reverse, 5'-CAATCTCACACTATCAAT CACATCGGAAT-3'; GAPDH forward, 5'-GCAAGTTCA ACGGCACAG-3' and reverse, 5'-CGCCAGTAGACTCCA CGAC-3'.

Statistical analysis. All statistical analyses were performed using SPSS software (version 11.0; SPSS, Inc. Chicago, IL, USA). Results are presented as the mean \pm standard error of the mean. Significant differences was estimated using one-way analysis of variance followed by Student-Newmann-Keuls multiple comparison tests. $\mathrm{P}<0.05$ was considered to indicate a statistically significant difference.

\section{Results}

Rat model of vascular calcification. Von Kossa staining results indicated that there was no calcification in the aorta of normal rats (Fig. 1A). However, there were strongly positively stained, black calcified nodules in the thoracic aorta of rats with vascular calcification (Fig. 1B), indicating the successful construction of the rat model of vascular calcification.

Decellularized arterial biological scaffold. There were numerous of nuclei both in normal arteries and calcified arteries (Fig. 2A and C; black arrows); however, no nuclei were found in the normal arterial decellularized scaffolds and calcified arterial decellularized scaffolds, and the elastic fiber structures were complete and arranged in an orderly manner (Fig. 2B and D; black arrows). Furthermore, there was no DNA band detected in the normal arterial decellularized scaffolds and calcified arterial decellularized scaffolds according to agarose gel electrophoresis results (Fig. 3), indicating the successful construction of decellularized biological scaffolds.

Expression of $\alpha$-SMA and $O P N$. The expression of $\alpha$-SMA mRNA was significantly increased in the normal decellularized arterial scaffold + VSMCs co-culture group compared with the calcified decellularized scaffold+VSMCs co-culture group on days 5, 10, 15 and 21 following establishment of the co-culture systems $(\mathrm{P}<0.05$ and $\mathrm{P}<0.001$; Fig. 4). Notably, the expression of OPN mRNA in the AR co-culture group was increased when compared with the normal co-culture group. This difference was statistically significant on days 15 and 21 following establishment of the co-culture systems $(\mathrm{P}<0.001$; Fig. 4). Western blot analysis revealed similar results. The results indicated that the phenotype of VSMCs was regulated by vascular calcification-induced AR may via downregulation of the expression of $\alpha$-SMA and upregulating the expression of OPN. 
A

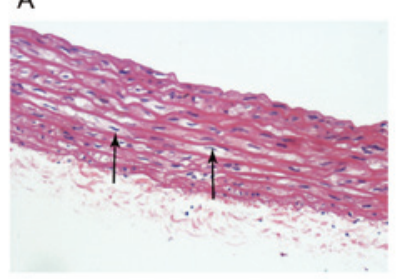

C

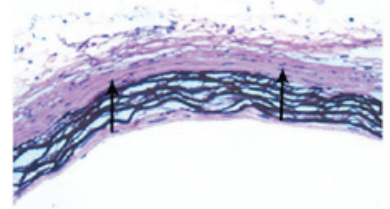

B

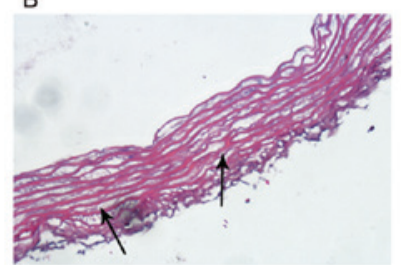

$\mathrm{D}$

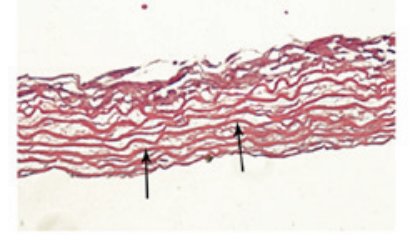

Figure 2. Biological scaffolds. (A) There were numerous nuclei in normal arteries (black arrows; magnification, $\mathrm{x} 40$ ). (B) There were no nuclei in the normal arterial decellularized scaffolds; however, the elastic fiber structure was complete and orderly arranged (black arrows; magnification, $\mathrm{x} 40$ ). (C) There were numerous nuclei in calcified arteries (white arrows; magnification, $x 40$ ). (D) There were no nuclei in the calcified arterial decellularized scaffolds, however, the elastic fiber structure was complete and orderly arranged (black arrows; magnification, x40).

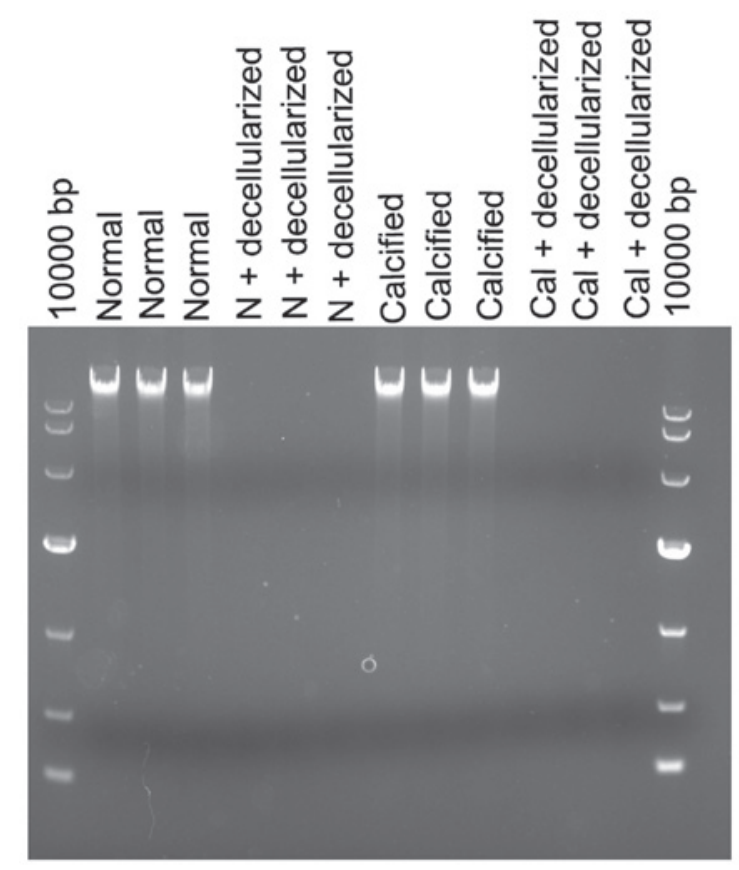

Figure 3. DNA band detection via agarose gel electrophoresis. There were DNA bands in both normal aortas and calcified aortas, while no DNA band was detected in the normal arterial decellularized scaffolds and calcified arterial decellularized scaffolds. Normal, normal aortas; N + decellularized, normal arterial decellularized scaffolds; Calcified, calcified aortas; Cal + decellularized, calcified arterial decellularized scaffolds.

\section{Discussion}

AR is a pathological manifestation of vascular disease complicated by hypertension, diabetes and other chronic diseases (7). AR is an important pathophysiological process in the development of vascular calcification (7). Notably, arterial calcification primarily occurs in the intima and middle membrane of the large artery and middle artery wall, which
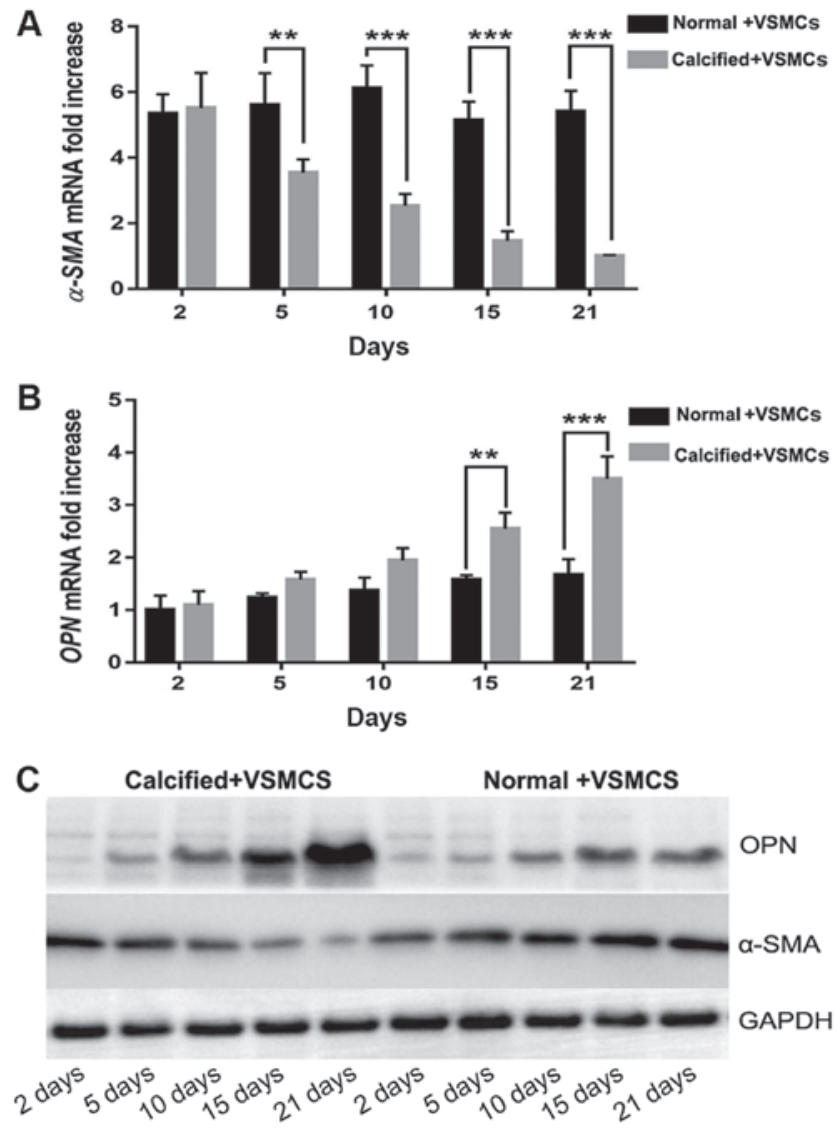

Figure 4. mRNA and protein expression of $\alpha$-SMA and OPN via reverse transcription-quantitative polymerase chain reaction and western blot analysis. (A) In the normal co-culture groups, the mRNA expression of $\alpha$-SMA was increased compared with the AR co-culture groups. (B) Furthermore, the mRNA expression of OPN in the AR co-culture groups was increased compared with the normal co-culture groups. (C) Western blot analysis indicated similar findings with regard to $\alpha$-SMA and OPN protein expression in the normal +VSMCs and calcified +VSMCs co-culture groups. ${ }^{* *} \mathrm{P}<0.05$ and ${ }^{* * *} \mathrm{P}<0.001$ as indicated. OPN, osteopontin; $\alpha$-SMA, $\alpha$-smooth muscle actin. Normal + VSMCs, normal arterial decellularized scaffolds + VSMCs co-culture group; Calcified + VSMCs, calcified arterial decellularized scaffolds + VSMCs co-culture group.

increases the probability of acute and chronic cardiac and cerebrovascular events, such as acute myocardial infarction, stroke, aneurysm rupture and bleeding, and seriously endangers the health and safety of individuals $(1,6)$. Therefore, an improved understanding of the pathogenesis of the AR process could provide useful information for the prevention and treatment of complications caused by vascular calcification. The AR process includes SMC migration and proliferation, and ECM production (7). However, little is known with regard to the changes that occur in ECM composition and SMCs during vascular calcification, even though $>50 \%$ of the neointimal hyperplasia consists of ECM proteins (7).

The mechanical properties of the ECM serve a critical role in regulating signaling pathways that underlie cell adhesion, migration, proliferation and differentiation $(18,19)$. Hypertension and other factors lead to changes in the proportion of ECM components in blood vessels, including an increase of collagen and fibrous adhesive protein, degradation of elastin and abnormal expression of matrix proteins, such as osteopontin (3), which lead to the thickening of the arterial 
wall, stenosis of the lumen and a decrease in vascular compliance (3). ECM is primarily composed of collagen and elastin and serves various functions that are essential for maintaining structural integrity of the cardiovascular wall (25). ECM remodeling is one of the underlying mechanisms in atherosclerotic cardiovascular disease, including as atherosclerosis, aneurysm formation and restenosis (7). The effect of ECM scaffolds in supporting tissue regeneration is mainly associated with two major characteristics: The maintained 3D structure and the bioactive components. The natural 3D structure of ECM provides structural support and tensile strength, attachment sites for cell surface receptors, and a reservoir for signaling factors that modulate angiogenesis, cell migration, cell proliferation, and orientation (26).

Elevated extracellular calcium and phosphate levels impact the survival and phenotype of VSMCs, which results in cellular adaptations and damage that ultimately promote calcification (8). As a result, the normal contractile VSMCs are converted into synthetic VSMCs (9), with significantly decreased $\alpha$-SMA expression and significantly increased OPN expression (10). The current experimental results were also consistent with that conclusion. In the current study, the results indicated that the expression of $\alpha$-SMA in the normal + VSMCs co-culture group was significantly increased, compared with the calcified + VSMCs co-culture group. $\alpha$-SMA is a specific phenotypic marker of VSMCs (27). In the current study, in the normal + VSMCs co-culture group, the scaffolds did not affect the $\alpha$-SMA expression of VSMCs. However, in the calcified + VSMCs co-culture group, the scaffolds reduced the expression of $\alpha$-SMA of VSMCs and this phenomenon was more pronounced with time. Furthermore, OPN is considered as a biomarker of VSMC phenotype switch and an active player in the progression of vascular remodeling diseases such as atherosclerosis (28). In the cureent study, the expression of OPN in the calcified + VSMCs co-culture group was significantly increased, compared with the normal + VSMCs co-culture group. The normal decellularized arterial scaffolds did not affect the expression of OPN in the normal + VSMCs co-culture group; while in the calcified + VSMCs co-culture group, the decellularized arterial scaffolds increased the expression of OPN, and this phenomenon was more pronounced with time.

To the best of our knowledge, the present study is the first to investigate the impact of normal and calcified decellularized arterial scaffolds on VSMCs. By constructing 3D arterial decellularized biological scaffolds and combining these with VSMCs, the impact of the changes in ECM on the VSMC phenotype could be studied. This study provides a novel platform for VSMC in vitro culture and associated disease mechanism research.

In a further study, the mechanisms of VSMC transformation induced by calcified arterial decellularized scaffolds and normal scaffolds will be investigated. Furthermore, the components and structures of the arterial decellularized biological scaffolds that can be used as possible therapeutic targets in vascular calcification will be explored.

In conclusion, arterial decellularized scaffolds may affect the transformation of VSMCs by downregulating the expression of $\alpha$-SMA and upregulating the expression of OPN. Future studies should be performed to investigate the overexpression of $\alpha$-SMA or knockdown of OPN to verify our hypothesis.

\section{Acknowledgements}

Not applicable.

\section{Funding}

The present study was supported by the Zhejiang Natural Science Foundation (grant no. Q15H170003) and Zhejiang Provincial Health Department Fund (grant no. 2017KY168).

\section{Availability of data and materials}

The datasets used and/or analyzed during the current study are available from the corresponding author on reasonable request.

\section{Authors' contributions}

LU and ZW wrote the first draft of the manuscript. HX studied the literature and conceived the study. SW, PW, XT and CX all performed in the experiments. All authors read and approved the final manuscript.

\section{Ethics approval and consent to participate}

The present study was approved by the Ethics Committee of The First People's Hospital of Taizhou.

\section{Patient consent for publication}

Not applicable.

\section{Competing interests}

The authors declare that they have no competing interests.

\section{References}

1. Demer LL and Tintut Y: Vascular calcification: pathobiology of a multifaceted disease. Circulation 117: 2938-2948, 2008.

2. Kay AM, Simpson CL and Stewart JA Jr: The role of AGE/RAGE signaling in diabetes-mediated vascular calcification. J Diabetes Res 2016: 6809703, 2016.

3. Pugliese G, Iacobini C, Blasetti Fantauzzi C and Menini S: The dark and bright side of atherosclerotic calcification. Atherosclerosis 238: 220-230, 2015.

4. Evrard S, Delanaye P, Kamel S, Cristol JP and Cavalier E; SFBC/SN Joined Working Group on Vascular Calcifications: Vascular calcification: From pathophysiology to biomarkers. Clin Chim Acta 438: 401-414, 2015.

5. Harper E, Forde H, Davenport C, Rochfort KD, Smith D and Cummins PM: Vascular calcification in type-2 diabetes and cardiovascular disease: Integrative roles for OPG, RANKL and TRAIL. Vascul Pharmacol 82: 30-40, 2016.

6. Lan TH, Huang XQ and Tan HM: Vascular fibrosis in atherosclerosis. Cardiovasc Pathol 22: 401-407, 2013.

7. Yahagi K, Kolodgie FD, Lutter C, Mori H, Romero ME, Finn AV and Virmani R: Pathology of human coronary and carotid artery atherosclerosis and vascular calcification in diabetes mellitus. Arterioscler Thromb Vasc Biol 37: 191-204, 2017.

8. Kapustin AN, Chatrou ML, Drozdov I, Zheng Y, Davidson SM, Soong D, Furmanik M, Sanchis P, De Rosales RT, Alvarez-Hernandez D, et al: Vascular smooth muscle cell calcification is mediated by regulated exosome secretion. Circ Res 116: 1312-1323, 2015. 
9. Alexander MR and Owens GK: Epigenetic control of smooth muscle cell differentiation and phenotypic switching in vascular development and disease. Annu Rev Physiol 74: 13-40, 2012.

10. Zhu LH, Huang L, Zhang X, Zhang P, Zhang SM, Guan H, Zhang Y, Zhu XY, Tian S, Deng K and Li H: Mindin regulates vascular smooth muscle cell phenotype and prevents neointima formation. Clin Sci (Lond) 129: 129-145, 2015.

11. Ruiz-Ortega M, Lorenzo O, Rupérez M, Esteban V, Suzuki Y, Mezzano S, Plaza JJ and Egido J: Role of the renin-angiotensin system in vascular diseases: Expanding thefield. Hypertension 38: $1382-1387,2001$.

12. Leask A and Abraham DJ: TGF-beta signaling and the fibrotic response. FASEB 18: 816-827, 2004.

13. Leask A, Holmes A and Abraham DJ: Connective tissue growth factor: A new and important player in the pathogenesis of fibrosis. Curr Rheumatol Rep 4: 136-142, 2002.

14. Amălinei C, Căruntu ID, Giuşcă SE and Bălan RA: Matrix metalloproteinases involvement in pathologic conditions. Rom J Morphol Embryol 51: 215-228, 2010.

15. Eweida AM and Marei MK: Naturally occurring extracellular matrix scaffolds for dermal regeneration: Do they really need cells? Biomed Res Int 2015: 839694, 2015.

16. Lindberg K and Badylak SF: Porcine small intestinal submucosa (SIS): A bioscaffold supporting in vitro primary human epidermal cell differentiation and synthesis of basement membrane proteins. Burns 27: 254-266, 2001.

17. Badylak SF, Taylor D and Uygun K: Whole-organ tissue engineering: Decellularization and recellularization of three-dimensional matrix scaffolds. Annu Rev Biomed Eng 15: 27-53, 2011.

18. Gessner RC, Hanson AD, Feingold S, Cashion AT, Corcimaru A, Wu BT, Mullins CR, Aylward SR, Reid LM and Dayton PA: Functional ultrasound imaging for assessment of extracellular matrix scaffolds used for liver organoid formation. Biomaterials 34: 9341-9351, 2013.
19. Ren H, Shi X, Tao L and Xiao J: Evaluation of two decellularization methods in the development of a whole-organ decellularized rat liver scaffold. Liver Int 33: 448-458, 2013

20. Sazonova OV, Isenberg BC, Herrmann J, Lee KL, Purwada A, Valentine AD, Buczek-Thomas JA, Wong JY and Nugent MA: Extracellular matrix presentation modulates vascular smooth muscle cell mechanotransduction. Matrix Biol 41: 36-43, 2015.

21. Butler DL, Goldstein SA and Guilak F: Functional tissue engineering: The role of biomechanics. J Biomech Eng 122: 570-575, 2000.

22. Steppan J, Bergman Y, Viegas K, Armstrong D, Tan S, Wang H, Melucci S, Hori D, Park SY, Barreto SF, et al: Tissue transglutaminase modulates vascular stiffness and function through crosslinking-dependent and crosslinking-independent functions. J Am Heart Assoc 6: pii: e004161, 2017.

23. Srivastava R, Zhang J, Go GW, Narayanan A, Nottoli TP and Mani A: Impaired LRP6-TCF7L2 activity enhances smooth muscle cell plasticity and causes coronary artery disease. Cell Rep 13: 746-13759, 2015.

24. Livak KJ and Schmittgen TD: Analysis of relative gene expression data using real-time quantitative PCR and the 2(-Delta Delta C(T)) method. Methods 25: 402-408, 2001.

25. Lacolley P, Regnault V, Segers P and Laurent S: Vascular smooth muscle and arterial stiffening: Relevance in development, aging, and disease. Physiol Rev 97: 1555-1617, 2017.

26. Badylak SF: The extracellular matrix as a scaffold for tissue reconstruction. Semin Cell Dev Biol 13: 377-383, 2002.

27. Han YL, Yan CH, Liu HW, Hu Y, Kang J, Wang X and Shaohua L: Overexpression of the cellular repressor of E1A-stimulated-genes regulate the rat primary VSMCs differentiation in vitro. Prog Biochem Biophys 31: 1099-1105, 2004.

28. Lee SJ, Baek SE, Jang MA and Kim CD: Osteopontin plays a key role in vascular smooth muscle cell proliferation via EGFR-mediated activation of AP-1 and C/EBP $\beta$ pathways. Pharmacol Res 108: 1-8, 2016. 